\title{
Comportamiento prosocial en una escuela chilena: Una intervención basada en la subjetividad del estudiantado ${ }^{1}$
}

\author{
Prosocial Behavior in a Chilean School: An Intervention Based on Student's Subjectivity²
}

\section{Comportamento pró-social numa escola chilena: uma intervenção baseada na subjetividade de estudantes ${ }^{3}$}

Recibido • Received • Recebido: 27 / 07 / 2018

Corregido • Revised • Revisado: 14 / 10 / 2019

Aceptado • Accepted • Aprovado: 17/ 02 / 2020

\begin{abstract}
Resumen: El objetivo de este trabajo fue describir e interpretar el proceso de cambio subjetivo, a partir de una intervención orientada a la reconstrucción de teorías subjetivas del comportamiento prosocial, en estudiantes de primer año de un liceo particular subvencionado de la comuna de Copiapó (Chile). En la intervención participó un curso de 45 estudiantes de primer año de educación secundaria y el cambio se evaluó mediante metodología cualitativa. Se aplicaron entrevistas episódicas y grupos de discusión y los datos se analizaron mediante codificación temática. Los resultados muestran que se produjo un cambio en el contenido y estructura de las teorías subjetivas de estudiantes, acerca del comportamiento prosocial, que orienta más funcionalmente la acción prosocial. En la discusión y conclusión se analiza el cambio subjetivo producido y se destaca la necesidad de continuar realizando estudios orientados a enriquecer la intervención para el desarrollo del comportamiento prosocial.
\end{abstract}

Palabras claves: Cambio subjetivo; comportamiento prosocial; estudiantes; adolescencia; educación socioemocional; teorías subjetivas.

\footnotetext{
${ }^{1}$ Esta investigación fue financiada por la Universidad de Atacama, a través del Fondo Concursable Dirección de Investigación, Proyecto DIUDA 22326.

${ }^{2}$ This research was funded by the University of Atacama, through the Concursable Fund Research Directorate, Project DIUDA 22326.

${ }^{3}$ Esta pesquisa foi financiada pela Universidade de Atacama, através da Diretoria de Pesquisa do Fundo Concursável, Projeto DIUDA 22326.
} 
http://doi.org/10.15359/ree.24-2.8

ROR: https://ror.org/01t466c14 Universidad Nacional, Costa Rica

http://www.una.ac.cr/educare

educare@una.cr

\begin{abstract}
This paper aims to describe and interpret the process of subjective change, based on an intervention aimed at the reconstruction of subjective theories of prosocial behavior, in secondary students of a subsidized private high school in Copiapó, Chile. Forty-five high school students participated in the intervention, and the effects of the intervention change were evaluated by using qualitative methodology. Episodic interviews and discussion groups were applied. Data was analysed through thematic coding. The results showed that there was a change in the structure and content of the subjective theories of students about prosocial behaviour, which was associated with greater functionality to behave prosocially. In the discussion, the subjective change produced was analysed. Likewise, the need to continue conducting studies aimed at enriching the intervention for the development of prosocial behaviour was highlighted.
\end{abstract}

Keywords: Subjective change; prosocial behaviour; students; adolescence; socioemotional education; subjective theories.

Resumo: $O$ objetivo deste trabalho foi descrever e interpretar o processo de mudança subjetiva, a partir de uma intervenção, orientada à pró-construção de teorias subjetivas do comportamento prósocial, nos estudantes do primeiro ano do ensino médio de uma escola particular da cidade de Copiapó (Chile). Desta intervenção participou uma turma de 45 estudantes do primeiro ano do ensino médio e a mudança foi avaliada mediante metodologia qualitativa. Foram aplicadas entrevistas episódicas e grupos de discussão. Os dados foram analisados mediante codificação temática. Os resultados mostram que houve mudança no conteúdo e na estrutura das teorias subjetivas dos estudantes sobre o comportamento pró-social, que orienta a ação pró-social de maneira mais funcional. Na discussão e conclusão foi analisada a mudança subjetiva produzida e se destacou a necessidade de continuar realizando estudos orientados a enriquecer a intervenção para o desenvolvimento do comportamento pró-social.

Palavras chave: Mudança subjetiva; comportamento pró-social; estudantes; adolescência; educação sócio emocional; teorias subjetivas.

\title{
Introducción
}

La prosocialidad es una de las respuestas a las actuales problemáticas de la convivencia social, dada su capacidad para resguardar el equilibrio social, constituirse en una alternativa para las conductas agresivas (Redondo et al., 2013), promover emociones positivas, asociarse con la resiliencia y mejorar la calidad de vida (Arias, 2015). También ha sido relacionada con el ajuste psicológico, por ejemplo, el autocontrol, el razonamiento moral (Lemos y Richaud de Minzi, 2010), la estabilidad emocional (Bekkers, 2006) y en contexto educativo, con un mejor desempeño académico (Inglés et al., 2013).

Sobre este comportamiento, actualmente se cuenta con una alta producción científica y con el interés de numerosas investigaciones (Einolf, 2010). Se define como aquellas acciones 
http://doi.org/10.15359/ree.24-2.8

ROR: https://ror.org/01t466c14 Universidad Nacional, Costa Rica http://www.una.ac.cr/educare

voluntarias que tienen como objetivo beneficiar a una o más personas, con conductas como la ayuda, la cooperación y el otorgar confort, teniendo a la base el altruismo (Pool, 2015).

Su delimitación conceptual es un debate que se encuentra en pleno proceso (Redondo et al., 2013), donde destacan posiciones que consideran, como núcleo de la definición, las condiciones cognitivo-motivacionales, versus aquellas que simplifican la manera de entenderlo, poniendo énfasis en la manifestación conductual de este comportamiento. Desde la primera corriente, lo que distingue a la prosocialidad de otros comportamientos positivos es la intensión de ayudar a otro a fin de beneficiarlo, lo cual se constituye como centro del concepto el altruismo; mientras que, en la segunda línea, el límite queda puesto en la manifestación de una conducta de ayuda, con independencia del tipo de intención o motivación.

La explicación del comportamiento prosocial ha derivado de un abanico de corrientes teóricas. Algunos estudios tienden a asociarlo con causas neuropsicológicas, con emociones positivas como la felicidad y la alegría (Coma y Carbonell, 2015); o al contrario, con emociones negativas frente a la proximidad de la muerte (Hirschberger et al., 2008), con componentes de la personalidad como la bondad humana y el altruismo (Warneken et al., 2011) o también con el logro y satisfacción personal, con arraigo en el egoísmo humano y los sentimientos de culpa (Einolf, 2010).

En contexto educativo, el desarrollo socioemocional del estudiantado resulta ser un desafío mayor, dado que las escuelas tienden a poner mayor énfasis en el aprendizaje de contenidos, privilegiando esto por sobre otro tipo de capacidades, no obstante la necesidad social de gestar el desarrollo de comportamientos que aporten a la sana convivencia, a la tolerancia y a la participación activa de la ciudadanía (Arias, 2015), sobre todo en América Latina, una de las regiones del mundo con mayor desigualdad. Lo anterior, porque la escuela constituye un espacio privilegiado para promover la prosocialidad, por la amplia red de interacciones que la caracterizan, los conflictos que presenta, las normas que la regulan, el desafío de interactuar con tolerancia, aceptación, democracia e inclusión (Redondo et al., 2013) y la posibilidad de implementar estrategias de enseñanza que integran la ayuda al otro ser, como el aprendizaje en servicio solidario.

Inglés et al. (2013) plantean que la prosocialidad predice las metas de aprendizaje, el logro estudiantil y el aprendizaje autónomo. En estudiantes adolescentes, este comportamiento adquiere una particular importancia, por encontrarse relacionado con una mejor adaptación social, un mayor rendimiento académico (Redondo et al., 2013), el establecimiento de relaciones de amistad, el éxito en las relaciones de pareja y la inclusión en grupos sociales con un estatus de mayor popularidad (Martínez et al., 2010). Por el contrario, las dificultades en las relaciones interpersonales en este período evolutivo se encuentran relacionadas con un alto número de alteraciones psicológicas y problemáticas educativas (Garaigordobil, 2014), por ejemplo, hay evidencia de que jóvenes con conducta antisocial muestran menor tendencia a considerar las 
http://doi.org/10.15359/ree.24-2.8

ROR: https://ror.org/01t466c14 Universidad Nacional, Costa Rica

http://www.una.ac.cr/educare

educare@una.cr

intenciones del otro individuo en procesos de interacción, lo que da cuenta de una dificulta importante para atender las características relevantes del contexto de interacción social (van den Bos et al., 2014).

Desde lo anterior, la intervención orientada al desarrollo del comportamiento prosocial en contextos educativos constituye una alternativa viable para la disminución de conductas violentas y la mejora del clima social escolar (Redondo et al., 2013). El trabajo de Boduszek et al. (2019), por ejemplo, mostró efectos significativos en la disminución de la violencia de género, a partir del uso de videojuegos prosociales en estudiantes de Barbados. Resultados similares se han obtenido en otros programas de intervención, en donde el desarrollo de la empatía y de la prosocialidad disminuyeron conductas violentas en estudiantes de educación primaria canadienses (Schonert-Reichl et al., 2012).

No obstante la importancia del desarrollo del comportamiento prosocial, los estudios orientados al diseño, implementación y evaluación de programas en esta área son menores (Ruiz, 2005). Algunos se han centrado en el juego cooperativo para promover la prosocialidad y prevenir la violencia en el estudiantado (Garaigordobil, 2014), el modelamiento, roleplaying y técnicas cognitivas conductuales; también en el desarrollo cognitivo y emocional, la identificación y solución de problemas sociales, el aprendizaje en servicio solidario y el incremento de las habilidades sociales (López et al., 2002).

Un factor significativo para el aprendizaje y desarrollo del comportamiento prosocial es el razonamiento internalizado o capacidad argumentativa, que posibilita comprender la situación en la cual se encuentra otra persona, anticipando consecuencias, evaluando el contexto interpersonal con base en atribuciones de justicia, lo que implica una toma de perspectiva y contrasta con aquellos razonamientos más hedonistas, que estarían a la base de un comportamiento más individualista, competitivo y egoísta. Para lo anterior, se requiere de una capacidad de razonamiento ante los problemas sociales (Mestre et al., 2006).

En este contexto, las creencias de la prosocialidad (Berger et al., 2016) regulan las interacciones sociales de ayuda y pueden orientar el comportamiento hacia la amabilidad, solidaridad y apoyo. De esta manera, para lograr desarrollar la empatía y la ayuda a otros seres, se requiere de creencias que permitan explicar el comportamiento de las personas, situación que se logra en un espacio de intersubjetividad.

Desde esta perspectiva, las representaciones subjetivas sobre la naturaleza humana y el ambiente, sobre todo del comportamiento altruista, adquieren elevada trascendencia (Ruiz, 2005). Lo que se ha encontrado es que adolescentes que manifiestan comportamientos de ayuda han reestructurado sus motivaciones personales producto de su participación en actividades de colaboración y apoyo voluntario; han incorporado en sus sistema de creencias las experiencias 
http://doi.org/10.15359/ree.24-2.8

ROR: https://ror.org/01t466c14 Universidad Nacional, Costa Rica http://www.una.ac.cr/educare

educare@una.cr

de voluntariado, y han llegado a elaborar metas prosociales (Cadena, 2010). También, quienes presentan creencias ecocéntricas tienden a tener un comportamiento de protección del medio social y natural (Miranda, 2013); su comportamiento de perdón se sustenta en teorías implícitas (Casullo, 2006) y estas últimas se relacionan con el comportamiento prosocial en estudiantes (Redondo et al., 2014).

Una forma de abordar la proconstrucción de la subjetividad de las personas es mediante la teoría del cambio subjetivo (Castro et al., 2015). En este modelo teórico las teorías subjetivas (TS) son definidas como un tipo particular de creencias elaboradas en la vida cotidiana, que tienen una estructura argumentativa y permiten explicar el propio comportamiento y el mundo, orientando en algún nivel la acción (Flick, 2006). LasTS incluyen un fuertecomponenteemocional, en la medida que se construyen a partir de la experiencia e interacción social, pudiendo ser una fuente de motivación y emociones positivas o, al contrario, inhibir los procesos de cambio y asociarse a emociones negativas que limitan el comportamiento humano. De esta manera, este modelo permite, en algún nivel, disminuir el alcance de enfoques más racionalistas, que abordan la comprensión y cambio del comportamiento humano.

Para el cambio de TS (Castro et al., 2015; Catalán, 2016; Cuadra-Martínez y Castro-Carrasco, 2018) se requiere abordar (a) el dominio emocional, a través de la identificación de la necesidad y motivación por el cambio, y es necesario explorar el contexto desde donde surgen y aplican estas explicaciones; (b) la explicitación y autoconocimiento de las TS, mediante métodos que permitan describirlas y posteriormente presentarlas a quienes las han elaborado; (c) el autoconocimiento y reconocimiento de las TS epistemológicas, que explican la disposición al propio proceso de cambio; (d) la reflexión sobre lasTS, como proceso central en la reconstrucción del propio pensamiento; (e) el trabajo grupal, desde donde es posible que los participantes contrasten, profundicen y evalúen sus propias formas de interpretar un fenómeno; (f) la presentación de la nueva información, que puede ser científica, valórica y de la vida cotidiana y representa una alternativa de enriquecimiento de las TS; y (g) las características del facilitador del cambio, básicamente referidas a la competencia y relación interpersonal humanista que establece con los beneficiarios.

La transformación de las TS implica un proceso de aprendizaje significativo, profundo, duradero y que permite reinterpretar el fenómeno objeto de enseñanza. A menudo, este proceso de cambio es evaluado como una transformación interna y radical, un aumento del sentido de eficacia respecto de la propia capacidad de aprendizaje y control sobre un fenómeno, una mayor funcionalidad, el otorgamiento de sentido a esta nueva forma de pensar, manifestado esto en una nueva TS que se caracteriza por una estructura argumentativa de mayor complejidad y variaciones en su contenido y significado (Catalán, 2016; Cuadra-Martínez y Castro-Carrasco, 2018). 
http://doi.org/10.15359/ree.24-2.8

ROR: https://ror.org/01t466c14 Universidad Nacional, Costa Rica

http://www.una.ac.cr/educare

educare@una.cr

Así, es posible afirmar que una intervención dirigida a estudiantes de educación secundaria, basada en la teoría del cambio subjetivo y orientada a la reconstrucción de TS del comportamiento prosocial, podría asociarse al desarrollo de un mayor comportamiento prosocial. Desde esto, surgen interrogantes: ¿de qué manera explican el comportamiento prosocial estudiantes de educación secundaria?, ¿cómo cambian estas explicaciones a partir de una intervención basada en el cambio subjetivo? y ¿qué factores se asocian a este proceso de cambio? Con base en esto, este trabajo buscó resolver el siguiente problema de estudio: Desde la perspectiva del estudiantado ¿cómo se produce el cambio subjetivo, a partir de una intervención orientada a la reconstrucción de teorías subjetivas del comportamiento prosocial, en estudiantes de primer año de enseñanza media de un liceo particular subvencionado? El objetivo general es describir e interpretar este proceso de cambio subjetivo, a partir de una intervención orientada a la reconstrucción de teorías subjetivas del comportamiento prosocial, en estudiantes de primer año de un liceo particular subvencionado chileno.

Respecto a la pertinencia y relevancia de este trabajo, es preciso señalar la importancia que tiene el aprendizaje y el desarrollo del comportamiento prosocial para la convivencia escolar, el éxito académico, la adaptación escolar (Inglés et al., 2013; Redondo et al., 2013), la salud mental y calidad de vida de la persona (Arias, 2015). Lo anterior, sobre todo en la adolescencia, por ser un período crítico en la configuración de la identidad personal y para el establecimiento de relaciones sociales positivas (Martínez et al., 2010). En este contexto, las TS representan una alternativa viable de abordaje, por ser un tipo de representación subjetiva de mayor elaboración y complejidad (Flick, 2006) y la subjetividad ser parte de una dimensión importante del comportamiento prosocial (Berger et al., 2016).

\section{Método}

\section{Tipo de estudio, metodología y diseño}

Corresponde a un estudio descriptivo interpretativo, que utiliza metodología cualitativa, pues lo que interesa es la realización de descripciones densas y profundas del fenómeno de estudio y desde la subjetivad de las personas (Flick, 2006). Se basa en un diseño de evaluación cualitativa, que permite evaluar el proceso de cambio subjetivo desde la perspectiva de los participantes.

La intervención se basó en la teoría del cambio subjetivo (Castro et al., 2015; Catalán, 2016; Cuadra-Martínez y Castro-Carrasco, 2018), específicamente, de la transformación de TS e incluyó 6 etapas que se describen en la Tabla 1.

6 
http://doi.org/10.15359/ree.24-2.8

ROR: https://ror.org/01t466c14 Universidad Nacional, Costa Rica

Tabla 1: Descripción de la intervención realizada

\begin{tabular}{ll}
\hline \multicolumn{1}{c}{ Etapas } & \multicolumn{1}{c}{ Descripción } \\
\hline Diagnóstico & $\begin{array}{l}\text { Aplicación de } 3 \text { grupos de discusión para la identificación de TS sobre el } \\
\text { comportamiento prosocial. }\end{array}$ \\
\hline Análisis de datos & Descripción de las TS sobre la prosocialidad. \\
\hline $\begin{array}{l}\text { Módulo 1: Abordaje de TS } \\
\text { epistemológicas }\end{array}$ & $\begin{array}{l}2 \text { sesiones de } 1^{1 / 2} \text { hora. para el autoconocimiento y reflexión sobre las TS } \\
\text { epistemológicas que el grupo posee (disposición al cambio y a la enseñanza y } \\
\text { el aprendizaje del tema), basado en dinámicas de grupo, exposición escrita y en } \\
\text { audio de las TS epistemológicas. }\end{array}$ \\
\hline Módulo 2: Abordaje de TS & $\begin{array}{l}2 \text { sesiones de } 1^{1 / 2} \text { hora. para el autoconocimiento y reflexión sobre las TS del } \\
\text { comportamiento prosocial (su desarrollo, definición y caracterización), basado } \\
\text { en dinámicas de grupo y análisis de casos en donde los participantes analizan } \\
\text { cómo sus TS orientan su comportamiento prosocial. }\end{array}$ \\
\hline Módulo 3: Presentación & $\begin{array}{l}1 \text { sesión de } 1^{1 / 2} \text { hora. orientada a que el estudiantado conozca, comprenda y } \\
\text { enjuicie información científica sobre la prosocialidad. Se basó en la presentación } \\
\text { de información en Power Point y guías de trabajo (definición, características, tipos, } \\
\text { factores que promueven y que inhiben la prosocialidad), el diálogo y la discusión. }\end{array}$ \\
información & $\begin{array}{l}\text { Aplicación de } 6 \text { entrevistas episódicas y } 3 \text { grupos de discusión para la } \\
\text { determinación del eventual cambio en las TS del comportamiento prosocial y } \\
\text { análisis cualitativo de datos. }\end{array}$ \\
\hline Evaluación &
\end{tabular}

Nota: Elaboración propia.

\section{Participantes}

Se trabajó con 45 estudiantes de primer año medio de un liceo particular subvencionado de la región de Atacama, Chile. El liceo se caracteriza por tener una matrícula para el año 2019 de 1209 estudiantes predominantemente de nivel socioeconómico medio. Imparte las modalidades técnico profesional y científico humanista.

Se seleccionó este curso a partir de las necesidades presentadas por la dirección de la escuela: corresponder a uno de los cursos con dificultades en la convivencia escolar, de esta manera el muestreo fue intencionado y por conveniencia (Flick, 2006).

Para la evaluación de la intervención, la muestra se definió considerando la asistencia a las sesiones de trabajo del taller, se excluyeron estudiantes que presentaron menos del $90 \%$ de asistencia al taller. De esta manera, se trabajó con un muestreo intencionado de 36 estudiantes, a quienes se aplicaron los instrumentos cualitativos. La edad promedio fue de 14 años; $60 \%$ fueron mujeres y $40 \%$ hombres. 
http://doi.org/10.15359/ree.24-2.8

ROR: https://ror.org/01t466c14 Universidad Nacional, Costa Rica

http://www.una.ac.cr/educare

educare@una.cr

\section{Procedimiento de recolección de información}

A través de la dirección de escuela se accedió a los sujetos participantes. Se realizó una reunión con estudiantes y sus padres y madres, a fin de explicar los fines de la investigación, la metodología de trabajo y los criterios éticos. Posteriormente, se aplicaron los instrumentos de recolección de información en instalaciones de la institución educativa. Se utilizaron dos instrumentos de recolección de datos, a fin de triangular la información y lograr una mayor credibilidad en el estudio.

Grupo de discusión: se aplicaron 3 grupos de discusión antes de la intervención y 3 al finalizar la intervención. Cada grupo quedó constituido por entre 6 y 10 participantes. En la investigación de TS, este instrumento ha sido indicado como una herramienta que permite contrastar y profundizar la información obtenida mediante entrevistas, posibilitando la reconstrucción deTS colectivas (Catalán, 2016). Los grupos de discusión se aplicaron considerando la técnica de entrevista episódica de Flick (2006), dado que se ha visto que es factible centrar la indagación de TS, con base en la elicitación de episodios biográficos en contexto grupal (Cuadra-Martínez y Castro-Carrasco, 2018). Técnicamente, quien entrevista debe (a) incentivar en los sujetos participantes la narración de situaciones y cadenas de situaciones relacionadas con el problema de estudio, (b) promover la generación de supuestos sobre el tema, (c) de definiciones subjetivas sobre el tema y (d) sus relaciones abstractas (Flick, 2006).

Antes de la intervención, el grupo de discusión se basó en el siguiente guion temático: (a) disposición al aprendizaje y desarrollo del comportamiento prosocial; (b) significado subjetivo del comportamiento prosocial; (c) explicaciones acerca del aprendizaje y desarrollo del comportamiento prosocial y (d) sugerencias para el desarrollo del comportamiento prosocial. Posterior a la intervención el guion temático consideró: (a) apreciaciones generales de la intervención, (b) eventual cambio producido, (c) tipo de cambio y (d) factores asociados al cambio. Tuvo una duración de entre 30 a 45 minutos y los datos se registraron en audio.

Entrevista episódica: esta entrevista permite acceder a un contenido narrativo-episódico y semántico, es especialmente indicada para la reconstrucción de TS (Flick, 2006). Las entrevistas se basaron en el mismo guion temático y la misma técnica utilizada en el grupo de discusión post intervención. Se aplicaron seis entrevistas episódicas al finalizar la intervención, a 6 estudiantes que se seleccionaron con el criterio de representatividad de casos, cautelando la participación de varones y mujeres, distinto rendimiento académico y nivel socioeconómico. Tuvo una duración aproximada de una hora y los datos se registraron en audio.

\section{Procedimiento de análisis de los datos}

Se transcribieron las entrevistas episódicas y grupos de discusión aplicados antes y después de la intervención. Los datos cualitativos obtenidos antes de la intervención permitieron orientar la intervención con base en las TS encontradas, mientras que los obtenidos al finalizar la intervención permitieron describir el cambio subjetivo producido. Los datos se analizaron 
http://doi.org/10.15359/ree.24-2.8

ROR: https://ror.org/01t466c14 Universidad Nacional, Costa Rica http://www.una.ac.cr/educare

educare@una.cr

mediante la técnica de codificación temática propuesta por Flick (2006). Esta técnica codifica la información textual en 2 etapas.

Codificación intracaso: durante esta fase, cada entrevista y grupo de discusión se analizó sintetizándolo como un caso y con un lema representativo del texto, una breve descripción del participante de la entrevista o grupo de discusión y los principales temas que caracterizan el texto (Flick, 2006). Cada investigador realizó el análisis intracaso por separado, para posteriormente llegar a construir una estructura conjunta y consensuada de este, lo que permitió una mayor credibilidad al estudio. Resultaron 12 casos codificados temáticamente.

Codificaciónintercaso:Losinvestigadoresanalizaronlos 12 casoscodificadostemáticamente, para llegar a elaborar un eje temático común a todos ellos (Flick, 2006). Este proceso lo realizaron conjuntamente. En este trabajo, en el apartado de resultados se presentan los principales temas reconstruidos y que hacen referencia al cambio producido por la intervención.

A continuación se muestran los resultados del estudio. Las citas textuales incluyen la siguiente simbología: $\mathrm{E}=$ entrevista; $\mathrm{GD}=$ grupo de discusión; $\mathrm{P}=$ participante.

\section{Resultados}

\section{Principales cambios en las teorías subjetivas}

\section{Tipo de cambio}

Desde la perspectiva de los participantes, los cambios producidos a partir de la intervención parecen ser a nivel actitudinal, situación que es advertida por el estudiantado como "una nueva forma de pensar". El estudiantado explica que en él se produjo (a) un mayor desarrollo conceptual de lo que es el comportamiento prosocial, (b) conocimiento procedimental de la conducta de ayuda, (c) una mayor disposición a ser prosocial o la confirmación del valor de su personalidad prosocial, (d) mayor conciencia de la relevancia de la reflexión para el comportamiento prosocial; (e) y del valor que tiene para el bienestar personal y el de otros sujetos.

El cambio a partir de la intervención también es advertido por el grupo a nivel de convivencia escolar. El estudiantado explica que, posterior a la intervención, el grupo curso desarrolló una mejor convivencia escolar, advertido esto en una mayor confianza, unión, cercanía y respeto entre estudiantes, y entre estudiantes y sus docentes. Así lo expresan algunos sujetos participantes:

Siento que hubo un cambio ahí, e, cambió mi modo de pensar, mi modo de socializar con las personas, hubo un cambio. (E4, mujer)

Por mi parte, lo principal fue saber cómo ayudar al otro o saber cuándo ayudar, eso es lo que más aprendí. (GD3, P6, hombre) 
http://doi.org/10.15359/ree.24-2.8

ROR: https://ror.org/01t466c14 Universidad Nacional, Costa Rica

http://www.una.ac.cr/educare

educare@una.cr

Ya no está tan disperso el curso. Al principio de año o por los primeros tres meses, se notaba que había muchos grupos separados. Ahora ya no se ve mucho eso... se están uniendo todos esos grupos en un curso. (GD2, P3, hombre)

Aprendí a ser más empática con la gente, a dar, a ayudar a la gente que antes no ayudaba, a dejar de ser tan individualista frente a algunas personas... estoy pensando más en el resto, que si a alguien le surge un problema, yo estoy dispuesta a ayudarla. (E1, mujer)

\section{Cambios en el significado y sentido de la prosocialidad}

Los participantes logran enriquecer el significado de prosocialidad, al desarrollar una definición más elaborada, además de asignarle un mayor valor a este tipo de comportamiento, así como a su enseñanza y aprendizaje, lo que se refleja en la reconstrucción de una TS más compleja, que orienta más funcionalmente la prosocialidad (Tabla 2).

Tabla 2: TS sobre el significado y sentido de la prosocialidad

\begin{tabular}{|c|c|c|c|}
\hline \multicolumn{2}{|c|}{ Antes de la intervención } & \multicolumn{2}{|c|}{ Después de la intervención } \\
\hline $\begin{array}{l}\text { TS reconstruida por el } \\
\text { investigador }\end{array}$ & Cita del sujeto participante & $\begin{array}{l}\text { TS reconstruida por el } \\
\text { investigador }\end{array}$ & Cita del sujeto participante \\
\hline $\begin{array}{l}\text { Ayudar o apoyar a otro } \\
\text { sujeto sin pedir nada a } \\
\text { cambio. Es antagónico al } \\
\text { egoísmo y a la maldad }\end{array}$ & $\begin{array}{l}\text { ¿Qué es el comportamiento } \\
\text { de ayuda? (Investigador). } \\
\text { Una persona ayudando a } \\
\text { otra (GD1, P2, mujer). } \\
\text { Beneficiar a alguien sin } \\
\text { pedir nada a cambio (GD2, } \\
\text { P8, hombre). }\end{array}$ & $\begin{array}{l}\text { Ayudar a otros voluntaria y } \\
\text { gratuitamente, para lograr } \\
\text { una mejor humanidad. La } \\
\text { prosocialidad se instala } \\
\text { sobre una actitud positiva, } \\
\text { promueve emociones } \\
\text { positivas y el crecimiento } \\
\text { personal y de otros. }\end{array}$ & $\begin{array}{l}\text { Teniendo más prosocialidad, va } \\
\text { a tener mayor empatía el curso, } \\
\text { va a ser más agradable, más } \\
\text { tolerante, más respetuoso (E1, } \\
\text { mujer). }\end{array}$ \\
\hline
\end{tabular}

Antes de la intervención

Después de la intervención

\section{TS reconstruida por el \\ Cita del sujeto participante} investigador

La prosocialidad, así como la enseñanza y aprendizaje de este comportamiento tiene regular valor.
¿Cómo está el ánimo para participar de este taller? No les veo con mucho convencimiento (Investigador).

No, no sé (risas), estoy dispuesta a escuchar no más (GD1, P3, mujer).
TS reconstruida por el investigador

La prosocialidad así como la Fue bueno porque aprendimos, enseñanza y aprendizaje de no sé, un mejor concepto este comportamiento tiene de la ayuda prosocial, nos un alto valor. pudimos conocer mejor como compañeros (GD3, P2, hombre).

A mí en lo personal, en el grupo en el que yo estoy nos llamó bastante la atención este taller, fuimos bastante participativos (E1, mujer).

Nota: Elaboración propia. 
http://doi.org/10.15359/ree.24-2.8

ROR: https://ror.org/01t466c14 Universidad Nacional, Costa Rica

\section{Cambios en las teorías subjetivas sobre el desarrollo de la prosocialidad}

Antes de la intervención, los sujetos participantes explican que el comportamiento prosocial se puede desarrollar a través de experiencias biográficas de ayuda a otras personas y también por medio de instancias de aprendizaje. Lo anterior, conduciría al desarrollo de una personalidad que tiene a la base valores altruistas que disponen a apoyar a quienes lo requieren. Los factores de mayor influencia lo representan (a) la familia, a través de la crianza y (b) los contextos sociales extraescolares. Para este alumnado, la educación formal tiene un menor impacto y en la medida que se progresa en nivel educativo, menor es la influencia. A la base, se observa una TS que asocia la prosocialidad con el desarrollo de la personalidad, a partir de experiencias familiares y extraescolares infantiles, lo que limita la disposición al aprendizaje de este comportamiento en otros períodos evolutivos y en la escuela. Posterior a la intervención, esta TS cambia, logrando mayor complejidad, incluye la educación formal como un factor importante de desarrollo de este comportamiento que dispone positivamente hacia el aprendizaje de la prosocialidad, en cualquier nivel evolutivo (Tabla 3).

Tabla 3: TS sobre el desarrollo de la prosocialidad

\begin{tabular}{|c|c|c|c|}
\hline \multicolumn{2}{|c|}{ Antes de la intervención } & \multicolumn{2}{|c|}{ Después de la intervención } \\
\hline $\begin{array}{l}\text { TS reconstruida por el } \\
\text { investigador }\end{array}$ & Cita del sujeto participante & $\begin{array}{l}\text { TS reconstruida por el } \\
\text { investigador }\end{array}$ & $\begin{array}{l}\text { Cita del sujeto } \\
\text { participante }\end{array}$ \\
\hline $\begin{array}{l}\text { La prosocialidad se } \\
\text { desarrolla a partir } \\
\text { de experiencias } \\
\text { biográficas familiares } \\
\text { y extraescolares } \\
\text { infantiles. Estas } \\
\text { experiencias inciden } \\
\text { en el desarrollo de una } \\
\text { personalidad prosocial }\end{array}$ & $\begin{array}{l}\text { ¿Se puede aprender a ser } \\
\text { prosocial? (Investigador). } \\
\text { Más que nada con la crianza } \\
\text { que nos dan nuestros familias y } \\
\text { los valores que nos dan desde } \\
\text { pequeños o pequeñas (GD2, P6, } \\
\text { hombre). } \\
\text { Es que el tema de ayudar } \\
\text { generalmente se aprende } \\
\text { cuando uno es chico, con la } \\
\text { mamá o el papá y ahora como } \\
\text { dijo el T., no se puede aprender } \\
\text { en esta edad, la personalidad de } \\
\text { uno ya está como... (GD1, P8, } \\
\text { mujer). }\end{array}$ & $\begin{array}{l}\text { La prosocialidad se } \\
\text { desarrolla a partir de } \\
\text { experiencias biográficas } \\
\text { familiares, sociales y } \\
\text { escolares. A través de } \\
\text { la educación formal se } \\
\text { puede desarrollar el } \\
\text { comportamiento prosocial } \\
\text { en cualquier período } \\
\text { evolutivo. }\end{array}$ & $\begin{array}{l}\text { ¿Un taller como este podría } \\
\text { ayudar a desarrollar la } \\
\text { prosocialidad en las personas? } \\
\text { (Investigador). } \\
\text { Sí, del curso, de las personas } \\
\text { en general yo creo que sí (E2, } \\
\text { hombre). } \\
\text { ¿Se puede aprender a ser } \\
\text { prosocial en la escuela? } \\
\text { (Investigador). } \\
\text { Si tienes las ganas, se puede } \\
\text { (GD3, P1, mujer). } \\
\text { Igual se puede aprender, ya } \\
\text { conmigo se puede, lo viví [en el } \\
\text { taller] (GD2, P5, hombre). }\end{array}$ \\
\hline
\end{tabular}

Nota: Elaboración propia. 
http://doi.org/10.15359/ree.24-2.8

ROR: https://ror.org/01t466c14 Universidad Nacional, Costa Rica

http://www.una.ac.cr/educare

educare@una.cr

\section{La enseñanza y aprendizaje de la prosocialidad}

En general, antes de la intervención los sujetos participantes presentan TS elaboradas acerca de la enseñanza y aprendizaje de la prosocialidad. Estas TS consideran que la prosocialidad se puede enseñar y aprender con base en (a) la experiencia biográfica, que implica vivir episodios en donde se haya practicado la prosocialidad, (b) el modelado de comportamientos prosociales, que involucra imitar a quienes son prosociales, (c) la enseñanza de valores como el respeto y la solidaridad y (d) la reflexión sobre la experiencia. Ahora, como se describió en el apartado anterior, estas instancias de aprendizaje se asocian menos con la educación formal y tienen efecto sobre todo en la infancia.

Posterior a la intervención, lo que fundamentalmente cambia es el desarrollo de una TS de mayor complejidad, que considera el efecto de la educación formal en el desarrollo de este comportamiento e incorpora conocimiento procedimental sobre cómo se enseña y aprende este tipo de comportamiento, en contextos de educación formal. Es decir, que al beneficiarse el estudiantado de una instancia de taller, esta experiencia le permite adquirir la capacidad de enjuiciar este espacio de trabajo y desde esto, sugerir herramientas, procedimientos y actividades concretas que podrían favorecer un mayor cambio en el comportamiento prosocial. Para estos grupos de estudiantes, las metodologías de enseñanza centradas en sus intereses y necesidades; y el trabajo grupal, práctico, aplicado y centrado en la reflexión son indicados para enseñar y aprender la prosocialidad en contextos educativos formales. La Tabla 4 muestra este proceso de cambio.

Tabla 4: TS sobre la enseñanza y aprendizaje de la prosocialidad

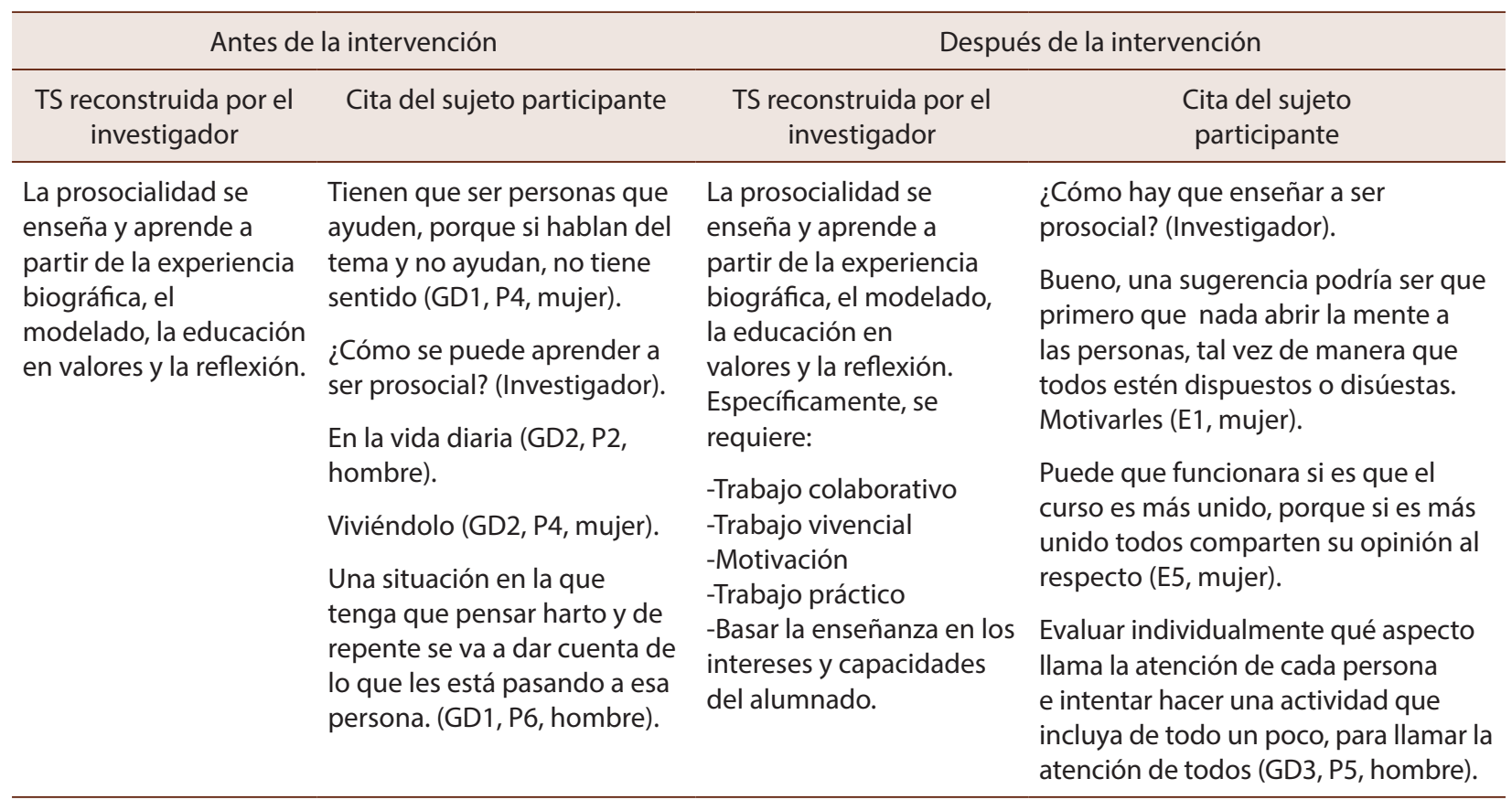

Nota: Elaboración propia. 
http://doi.org/10.15359/ree.24-2.8

ROR: https://ror.org/01t466c14 Universidad Nacional, Costa Rica

\section{Factores asociados al cambio subjetivo}

El grupo de participantes identifica una serie de factores asociados al cambio subjetivo. Es posible clasificarlos como (a) condiciones cognitivo-emocionales de los aprendices y (b) el motor de cambio de la intervención.

\section{Condiciones cognitivo-emocionales de los sujetos aprendices}

Desde el estudiantado, se requiere de un sentido de autoeficacia adecuado para el cambio subjetivo y cuando lo que se busca es el aprendizaje de la prosocialidad. Explican que frente a este tipo de temáticas, quienes consideran incorrectamente poseer un adecuado dominio y saber sobre el tema, podrían inhibir su motivación o disposición al cambio. En una interpretación de segundo orden, esto podría ocurrir sobre todo frente a un tema que se encuentra más ligado al saber subjetivo o de la vida cotidiana, que tiene un fuerte arraigo y ha demostrado serles funcional.

La motivación por el aprendizaje o cambio también es señalada como un factor crítico para el cambio, en el contexto de un taller de desarrollo de la prosocialidad. Desde sus TS, hay condiciones personales y propias de la intervención que se asocian a una mayor o menor motivación.

Finalmente, la capacidad de reflexión es indicada como un factor clave que posibilita contrastar experiencias, analizar las emociones propias y la forma de pensar, enjuiciar el propio comportamiento y la nueva información, y concluir sobre nuevas formas de pensar o saber. La Tabla 5 describe más específicamente los factores de cambio advertidos por los sujetos participantes.

Tabla 5: La autoeficacia, motivación y reflexión como factores de cambio subjetivo

\begin{tabular}{|c|c|c|}
\hline \multicolumn{3}{|c|}{ Después de la intervención } \\
\hline TS reconstruida por el investigador & Característica de la TS & Cita del sujeto participante \\
\hline $\begin{array}{l}\text { El cambio subjetivo, en contexto } \\
\text { de enseñanza y aprendizaje de } \\
\text { la prosocialidad depende de } \\
\text { condiciones cognitivo-emocionales } \\
\text { de los aprendices como: } \\
\text {-Un adecuado sentido de } \\
\text { autoeficacia } \\
\text { - Motivación por el aprendizaje o } \\
\text { cambio. } \\
\text { - Reflexión. }\end{array}$ & $\begin{array}{l}\text { Estructura compleja. } \\
\text { Se asocia a un pensamiento } \\
\text { superior (aprender a aprender), } \\
\text { que permite explicar cómo } \\
\text { producir un cambio subjetivo } \\
\text { cada persona en sí misma o en } \\
\text { otras. }\end{array}$ & $\begin{array}{l}\text { ¿En un taller como este, qué puede hacer que las } \\
\text { personas cambien respecto de la prosocialidad? } \\
\text { (Investigador). } \\
\text { La experiencia, razonar, pensar (E2, hombre). } \\
\text { No sé, como que reflexionamos lo que tenemos, } \\
\text { por ser que tenemos todas las partes del cuerpo y } \\
\text { ayudar a las personas. (GD3, P9, hombre). } \\
\text { Poner atención y poner de mi parte, porque si no } \\
\text { pongo de mi parte no llego a nada (E3, hombre). } \\
\text { [Algunos no aprenden] Porque no les interesa } \\
\text { mucho esto o porque ya saben lo suficiente o creen } \\
\text { que saben lo suficiente, pero no saben (E4, mujer). }\end{array}$ \\
\hline
\end{tabular}

Nota: Elaboración propia. 
http://doi.org/10.15359/ree.24-2.8

ROR: https://ror.org/01t466c14 Universidad Nacional, Costa Rica

http://www.una.ac.cr/educare

educare@una.cr

\section{Características de la intervención}

Los sujetos participantes asocian una serie de factores que son constituyentes de una intervención orientada al cambio de TS, en este caso, de la prosocialidad. Además, este grupo de estudiantes logra contrastar este tipo de trabajo, con otras metodologías de enseñanza y aprendizaje en las que han participado, reconociendo particularidades de una intervención orientada al cambio subjetivo, así como su potencial para favorecer el cambio. Así lo expresan:

En primer lugar, me pareció muy novedoso, porque en mi anterior escuela hacíamos talleres, pero nunca se enmarcaba en la prosocialidad. Siempre era como para presentarnos y se terminaba. Tal vez me ayudó a darme nuevas perspectivas. (E3, hombre)

Fue muy como entretenido, así muy llamativo, aprendimos cosas nuevas y el significado de empatía. Nos daba qué pensar o analizar situaciones por nosotros mismos. (E2, hombre)

Fue bueno, al principio de año no estábamos unidos cuando llegaron los talleres. Es bueno tener un taller con psicólogos que te abren la mente a otras opciones. (E4, mujer)

Específicamente, los factores asociados al cambio de TS son: el (a) favorecimiento de la interacción social y el conocimiento del otro individuo, lo que permite contrastar las distintas formas de interpretar fenómenos, además de generar confianza entre aprendices y el enriquecimiento las TS. También es necesario (b) generar espacios de expresión emocional y de manifestación de comportamientos propositivos, porque implica hacer un cambio en la forma habitual de comunicarse y produce respuestas de reciprocidad. El considerar (c) la experiencia biográfica es un factor clave, porque favorece el aprendizaje desde el testimonio de pares y permite otorgarle sentido al conocimiento objetivo, que a menudo es caracterizado como abstracto y poco aplicable. Además, se señala que hay (e) características que debe presentar quien interventa, tales como competencia en lo que enseña, paciencia, comprensión, una relación de confianza y cercanía. Finalmente, el (f) tomar conciencia del cambio, confirmarlo y evaluarlo es indicado como una estrategia que permite arraigar lo aprendido (Tabla 6).

\section{Discusión}

El objetivo de este estudio fue describir e interpretar, desde la perspectiva del estudiantado, cómo se produce el cambio subjetivo, a partir de una intervención orientada a la reconstrucción de teorías subjetivas del comportamiento prosocial, en estudiantes de primer año de enseñanza media de un liceo particular subvencionado. 
http://doi.org/10.15359/ree.24-2.8

ROR: https://ror.org/01t466c14 Universidad Nacional, Costa Rica

Tabla 6: Factores de la intervención que favorecen el cambio subjetivo

\begin{tabular}{|c|c|}
\hline Factor & Cita del sujeto participante \\
\hline \multirow[t]{2}{*}{$\begin{array}{l}\text { Interacción social y } \\
\text { conocimiento del otro ser }\end{array}$} & $\begin{array}{l}\text { Empezar interactuar más con la gente, que antes no era de mi entorno. } \\
\text { Comunicarme más con la gente, porque antes yo tenía mi grupito y no hablaba con } \\
\text { el resto, porque me caían mal, porque pensaban diferente a mí, entonces esto me } \\
\text { ayudó a ser un poco más empática con el resto (E3, hombre). }\end{array}$ \\
\hline & $\begin{array}{l}\text { A mí me hizo sentir bien, o sea saber lo que esa persona pensaba de mí por mis actos, } \\
\text { o sea por ejemplo, si yo lo ayudo, y lo hago sentir bien y él me devuelve, o sea me } \\
\text { dieron más ganas de ayudar a las otras personas si eso les hace bien (GD3, P7, mujer). }\end{array}$ \\
\hline \multirow{3}{*}{$\begin{array}{l}\text { Expresión emocional } \\
\text { y manifestación de } \\
\text { comportamientos } \\
\text { propositivos }\end{array}$} & $\begin{array}{l}\text {... yo lo destaco porque uno al agradecerle cosas a las otras personas, la otra persona } \\
\text { se siente bien y le dan más ganas de seguir con la ayuda prosocial (GD3, P6, hombre). }\end{array}$ \\
\hline & $\begin{array}{l}\text { El último taller, donde nos hizo agradecerle a las personas, me pareció muy } \\
\text { interesante, porque como que uno nunca, como que nunca agradece lo suficiente } \\
\text { a las personas que nos hacen un bien. Porque yo por ejemplo, nunca he sido muy } \\
\text { agradecida con mi mamá. Nunca agradezco lo que ella hace por mí (E3, hombre). }\end{array}$ \\
\hline & Poner las cosas positivas del curso, la de nuestros compañeros (E4, mujer). \\
\hline Experiencia biográfica & $\begin{array}{l}\text { La experiencia sentimental y que a través de una experiencia uno pueda sentirse } \\
\text { más en el papel y decir, "a, sí tiene razón” (E2, hombre). }\end{array}$ \\
\hline $\begin{array}{l}\text { Conciencia, confirmación } \\
\text { y evaluación del cambio }\end{array}$ & $\begin{array}{l}\text { Me llamó la atención la primera clase, la entrevista, fue la introducción de todo } \\
\text { lo que íbamos a ver en el semestre. Y después ahora mismo, después de esa } \\
\text { introducción, todo lo que aprendieron, a partir de esa introducción. Esto sirve para } \\
\text { saber lo que van a ver durante todas estas clases... y ahora estamos hablando sobre } \\
\text { todo lo que aprendimos durante este semestre (GD3, P2, hombre). }\end{array}$ \\
\hline \multirow{4}{*}{$\begin{array}{l}\text { Con intervención } \\
\text { competencia, paciencia, } \\
\text { comprensión y que } \\
\text { genera confianza }\end{array}$} & ¿Qué otro elemento permitió el cambio en su forma de pensar? (Investigador). \\
\hline & La paciencia (GD3, P4, mujer). \\
\hline & $\begin{array}{l}\text { Ser amigable, sentirse como igual al profesor, una relación de más confianza, eso } \\
\text { ayudaba a comprender más (GD3, P7, mujer). }\end{array}$ \\
\hline & $\begin{array}{l}\text { Cuando explicaban las actividades las explicaban bien y tenían harta paciencia } \\
\text { (GD3, P8, hombre). }\end{array}$ \\
\hline
\end{tabular}

Nota: Elaboración propia.

Mediante la intervención realizada, fue posible generar un cambio de TS de la prosocialidad. Estudios similares señalan que la transformación de la subjetividad a la base del comportamiento prosocial se asocia con una regulación de las interacciones sociales de ayuda, solidaridad, apoyo (Berger et al., 2016), orientación hacia la amabilidad (Casullo, 2006), construcción de metas prosociales (Cadena, 2010) y creencias eco-céntricas (Miranda, 2013). 
http://doi.org/10.15359/ree.24-2.8

ROR: https://ror.org/01t466c14 Universidad Nacional, Costa Rica

http://www.una.ac.cr/educare

educare@una.cr

Así, este trabajo podría aportar a la planificación e implementación de intervenciones psicológicas educativas orientadas al desarrollo del comportamiento prosocial en el estudiantado, dado el insuficiente diseño, implementación y evaluación de programas en esta área (Ruiz, 2005).

El estudiantado advierte una transformación no solo en su forma de pensar respecto de la prosocialidad, sino que además identifica una mayor disposición para comportarse prosocialmente y una mayor capacidad. En contexto educativo, otros estudios han señalado que la intervención orientada a la transformación de las TS es experimentada por los sujetos beneficiarios como un cambio en la forma de pensar, un aumento del sentido de autoeficacia y control sobre un fenómeno y una mayor funcionalidad (Castro et al., 2015).

Parte de esta transformación de TS se presentó en el significado y valor atribuido a la prosocialidad. Básicamente, el estudiantado logró desarrollar una TS de mayor complejidad, que implica el establecimiento de nuevas conexiones de información y una mayor riqueza semántica.

La complejidad de una TS ha sido relacionada con una mayor capacidad de reflexión, un mayor autoconocimiento y comprensión de un fenómeno, lo que permite abordarla más eficientemente (Catalán, 2016; Waeytens, Lens y Vandenberghe, 2002). Esto parece ser particularmente importante en el desarrollo de la prosocialidad, porque este comportamiento requiere de una capacidad para razonar y argumentar, para comprender y tomar decisiones en un contexto social en que se requiere de ayuda (Mestre et al., 2006).

También se observó una mayor valoración de la prosocialidad y de la enseñanza y aprendizaje de este comportamiento, lo que es un cambio clave porque, en general, las escuelas tienden a no valorar suficientemente el desarrollo socioemocional de sus estudiantes y han puesto mayor énfasis en el aprendizaje de información formal. Esto podría explicar por qué este grupo de estudiantes, antes de la intervención, le atribuyó un mínimo valor a la prosocialidad y un bajo impacto a la educación formal, en el desarrollo de este comportamiento. Este cambio hace referencia a la dimensión emocional de lasTS, ámbito que es fundamental para el desarrollo de la conducta prosocial.

Ello parece fundamental, porque la disposición y motivación por el cambio subjetivo ha sido indicado como un factor promotor de este (Smith et al., 2009). En esta intervención, se consideró inicialmente la indagación de las TS epistemológicas (Cuadra-Martínez y CastroCarrasco, 2018), porque este tipo de explicaciones enjuician el conocimiento que se pretende enseñar, incluyendo en su estructura argumentativa una evaluación de su valor o utilidad, que puede ser positiva o negativa. Precisamente, este tipo de TS determinan la disposición ante el cambio. Fussangel (2008) señala que es necesario identificar el contexto en donde aplican las TS, porque la motivación por el cambio subjetivo se presenta cuando el sujeto participante somete a prueba sus TS en otros contextos. En este taller, mediante los grupos de discusión, se identificaron las TS acerca de la importancia y pertinencia de enseñar y aprender 
sobre prosocialidad, y se encontró que estas tendían a inhibir su disposición a participar de un proceso de cambio subjetivo. Así, fue necesario que a través de actividades de estudio de casos y análisis de experiencias biográficas propias y de sus pares, sometieran a prueba la funcionalidad de mantener este tipo de explicaciones. En otros modelos de intervención que también han mostrado resultados positivos, el desarrollo de la prosocialidad se ha centrado en la identificación y solución de problemáticas sociales (López et al., 2002), lo que da luces de la importancia de considerar contextos reales de aplicabilidad, del conocimiento aprendido, en donde se ponga a prueba el saber subjetivo.

El cambio subjetivo producido mediante esta intervención, y desde la perspectiva de los sujetos participantes, también implicó el desarrollo de un pensamiento superior, específicamente, una mayor capacidad de aprender a aprender o de aprendizaje autorregulado. En este caso, tras la intervención, cada participante elaboró una TS que no solo dispone a ser prosocial y explica, desde cada quien, de mejor forma, el desarrollo de este comportamiento y su manifestación, sino que además refiere de manera procedimental cómo se podría aprender y enseñar a ser prosocial. En otros trabajos también se ha encontrado que la prosocialidad se relaciona con las metas de aprendizaje y el aprendizaje autorregulado (Inglés et al., 2013).

Desde esto, es sugerente iniciar nuevas investigaciones orientadas a determinar cómo el cambio subjetivo de TS se inserta con la capacidad para autorregular el aprendizaje, puesto que esta última capacidad ha sido relacionada con la autoeficacia, implicancia, la motivación, control y autonomía en el propio aprendizaje (Altun y Erden, 2013).

En relación con lo anteriory desde los participantes, una intervención orientada al desarrollo de la prosocialidad podría considerar como factores promotores del cambio subjetivo a: (a) la motivación por el cambio y por el aprendizaje y enseñanza de este tipo de comportamiento; (b) el favorecimiento de la interacción social, ya que actúa como un espacio de intersubjetividad en donde se evalúa y pone a prueba la prosocialidad; (c) la reflexión sobre el propio pensamiento y comportamiento prosocial; (d) la expresión emocional y propositividad, que actúan como transformadoras del comportamiento individual y social; (e) y el relacionar lo que se aprende con la propia experiencia, porque permite otorgar significado y sentido al nuevo contenido y, en este contexto, el uso de testimonios puede ser una tarea concreta que facilita este trabajo.

El sentido de autoeficacia es un factor que en otros trabajos ha sido señalado como indicador de cambio subjetivo (Catalán, 2016; Cuadra-Martínez y Castro-Carrasco, 2018). En este trabajo, además, se relaciona con la disposición y motivación por el cambio. Desde los sujetos participantes, un muy alto sentido de autoeficacia en la tarea podría representar un exceso de confianza en el dominio de una tarea, conllevando esto a desvalorar o considerar innecesario participar en un contexto de enseñanza y aprendizaje. Esto también podría explicar la reducida valoración de la enseñanza y aprendizaje de la prosocialidad del alumnado antes de la intervención. Sin embargo, contrasta con la vasta bibliografía que señala que las creencias de 
http://doi.org/10.15359/ree.24-2.8

ROR: https://ror.org/01t466c14 Universidad Nacional, Costa Rica

http://www.una.ac.cr/educare

educare@una.cr

autoeficacia se relacionan directamente con la motivación por una tarea (Khalid, 2014). Podría ser que la dificultad percibida ante una tarea sea una variable mediadora entre la autoeficacia y la motivación académica, lo que estaría en línea con lo propuesto por Oyserman (2015): que la dificultad percibida ante una tarea regula el nivel de importancia que se le atribuye a esta. Así, tareas evaluadas como demasiado fáciles, o demasiado difíciles adquieren un menor valor para la construcción de la identidad y, por lo tanto, son menos motivantes.

Es necesario, por lo tanto, continuar indagando cómo se relaciona el cambio subjetivo de la prosocialidad, con el sentido de autoeficacia y la dificultad percibida ante una tarea. Sobre todo cuando el tema de aprendizaje se encuentra más relacionado con el saber subjetivo o de la vida cotidiana, porque este tipo de conocimiento tiene un fuerte arraigo y ha demostrado ser funcional. Probablemente esto puede ocurrir con aquellos contenidos no formales, ligados al desarrollo de competencias genéricas, como la prosocialidad.

Desde este trabajo surge la hipótesis de que un exceso de confianza en lo que se sabe, probablemente orienta a la ejecución de una tarea, pero no siempre a continuar aprendiendo "o perfeccionándose" en el tema. En el modelo de cambio de TS, la propuesta es someter a prueba las explicaciones que se poseen sobre el tema en cuestión, en otros contextos de aplicabilidad en donde estas TS no son suficientes para responder adecuadamente, para que quien aprende tome conciencia de la necesidad de disponerse a enriquecer su forma de pensar, para lograr una mayor funcionalidad (Fussangel, 2008).

Para finalizar, se señala que también es importante continuar investigando cuál podría ser la didáctica más pertinente para lograr promover, en grupos adolescentes, procesos reflexivos acerca de la prosocialidad, a propósito de que la reflexión ha sido considerada como el principal factor promotor del cambio subjetivo (Catalán, 2016). En este contexto, en la adolescencia se requiere de tareas distintas a las de las personas adultas, y los procesos reflexivos incluso parecen indicar distintos tiempos para realizarlo. En esta intervención, una conclusión es que los grupos de adolescentes requieren de una mayor relación entre las tareas prácticas vivenciales - reales y las tareas de nivel más abstracto; además de procesar y representar una misma información de múltiples formas, por ejemplo, por medio de imágenes, palabras, juegos y mediante información metafórica, biográfica y formal.

Son necesarias nuevas investigaciones en esta línea, para continuar precisando qué tareas podrían ser las más indicadas para favorecer el cambio subjetivo con adolescentes, sobre todo en el ámbito de la educación socioemocional y la prosocialidad, por ser este un comportamiento altamente relacionado con la salud mental y el adecuado ajuste social (Arias, 2015; Bekkers, 2006; Inglés et al., 2013; Lemos y Richaud de Minzi, 2010; Redondo et al., 2013). También es necesario señalar que se necesitan nuevas investigación, en lo sucesivo cuantitativas y con variados diseños, que permitan aportar más evidencia empírica acerca de cómo la reconstrucción de teorías subjetivas sobre la prosocialidad puede constituirse en un aporte para el desarrollo del comportamiento prosocial en estudiantes. 
http://doi.org/10.15359/ree.24-2.8

ROR: https://ror.org/01t466c14 Universidad Nacional, Costa Rica http://www.una.ac.cr/educare

educare@una.cr

\section{Conclusión}

El desarrollo, aprendizaje y enseñanza del comportamiento prosocial en contextos educativos representa una alternativa importante para que el estudiantado logre una adecuada adaptación escolar y social, y la comunidad educativa promueva una convivencia escolar positiva.

En este trabajo se presentó una intervención de cambio subjetivo, basada en la transformación de las TS que poseen los grupos de estudiantes, acerca de la prosocialidad. La evaluación de la intervención evidenció que podría ser factible implementar este tipo de trabajo en estudiantes de educación secundaria, no solo porque favorece el desarrollo de un pensamiento prosocial, sino porque, además, los sujetos participantes valoran este tipo de instancia como una forma motivante y distinta de aprender sobre el tema.

De esta manera, puede representar una guía para que personal docente, orientador o profesional del área de las ciencias sociales aborde la enseñanza y aprendizaje de la prosocialidad en contexto educativo. Básicamente, quienes opten por un trabajo como este, deben considerar que las herramientas favorecedoras del cambio apuntan a que el estudiantado conozca y enjuicie sus propias explicaciones acerca del tema en cuestión, sobre todo si esta forma de pensar favorece o no la motivación por el cambio. Esto permite flexibilizar el pensamiento y disponerse a aprender la nueva información o el contenido sobre el tema. Para esto, se requiere de trabajo grupal, reflexión y procesamiento de la información mediante múltiples canales de comunicación.

En cuanto a los aportes teóricos, el trabajo expuesto contribuye a la teoría del comportamiento prosocial, básicamente nutriendo el modelo de creencias prosociales, señalando el rol que juegan las TS en este tipo de comportamiento y exponiendo evidencia empírica acerca de cómo es que se transforman este tipo de representaciones cognitivas.

Finalmente, surgen interrogantes y desafíos necesarios de abordar en futuras investigaciones. La primera de ellas es sobre la sustentabilidad del cambio valorado desde un enfoque longitudinal. Diseños mixtos y longitudinales podrían ser indicados para resolver esta interrogante. También se requieren estudios que permitan cuantificar el cambio subjetivo logrado y su relación con las prácticas prosociales. La segunda, es más específica y se refiere a la relación entre las creencias de autoeficacia, el grado de dificultad percibido ante una tarea y la disposición al cambio subjetivo o aprendizaje. Desde este trabajo, la relación entre estas variables parece ser clave para la transformación de las TS. Sin embargo, no queda del todo claro cómo es que se relacionan, por lo que se requiere de otros estudios que aporten en comprensión y, de esta manera, a la teoría del cambio subjetivo en contexto educativo. 
http://doi.org/10.15359/ree.24-2.8

ROR: https://ror.org/01t466c14 Universidad Nacional, Costa Rica

http://www.una.ac.cr/educare

educare@una.cr

\section{Referencias}

Altun, S.y Erden, M. (2013). Self-regulation based learning strategies and self-efficacy perceptions as predictors of male and female student's mathematics achievement. Procedia - Social and Behavior Sciences, 106, 2354-2364. https://doi.org/10.1016/j.sbspro.2013.12.270

Arias Gallegos, W. (2015). Conducta prosocial y psicología positiva. Avances en Psiclogía., 23(1), 37-47. https://doi.org/10.33539/avpsicol.2015.v23n1.169

Bekkers,R.(2006).Traditionaland health related philanthropy:Theroleofresourcesand personality. Social Psychology Quarterly, 69(4), 349-366. https://doi.org/10.1177/019027250606900404

Berger, C., Cuadros, O., Rasse, C. y Rojas, N. (2016). Diseño y validación de la escala de creencias normativas sobre la prosocialidad en adolescentes chilenos. Psykhe, 25(1), 1-17. https:// doi.org/10.7764/psykhe.25.1.692

Boduszek, D., Debowska, A., Jones, A. D., Ma, M., Smith, D., Willmott, D., Trotman Jemmott, E., Da Breo, H. y Kirkman, G. (2019). Prosocial video game as an intimate partner violence prevention tool among youth: A randomised controlled trial. Computers in Human Behavior, 93, 260-266. https://doi.org/10.1016/j.chb.2018.12.028

Cadena, M. P. (2010). Aportes y desafíos de estudiar el voluntariado desde la mirada del sujeto: Análisis de los hallazgos de Marta, Pozzi y Marzana (2010). Psykhe, 19(2), 19-23. https://doi. org/10.4067/S0718-22282010000200003

Castro, P. J., Krause, M. y Frisancho, S. (2015). Teoría del cambio subjetivo: Aportes desde un estudio cualitativo con profesores. Revista Colombiana de Psicología, 24(2), 363-379. https://doi.org/10.15446/rcp.v24n2.44453

Casullo, M. M. (2006). Las razones para perdonar. Concepciones populares o teorías implícitas. Psicodebate, 7, 9-20. https://doi.org/10.18682/pd.v7i0.424

Catalán, J. (2016). Hacia la formulación de una teoría general de las teorías subjetivas. Psicoperspectivas. Individuo y Sociedad, 15(1), 53-65. https://doi.org/10.5027/ psicoperspectivas-Vol15-Issue1-fulltext-739

Coma, J. y Carbonell, J. (2015). Experiencia de libre albedrío en la conducta pro-social determinada por la alegría. Escritos de Psicología, 8(3), 41-47. https://doi.org/10.5231/psy.writ.2015.3007

Cuadra-Martínez, D. y Castro-Carrasco, P. (2018). Cambio educativo: Prouesta de un modelo de transformación de teorías subjetivas. Liberabit, 24(1), 147-164. https://doi.org/10.24265/ liberabit.2018.v24n1.10 
http://doi.org/10.15359/ree.24-2.8

ROR: https://ror.org/01t466c14 Universidad Nacional, Costa Rica http://www.una.ac.cr/educare

Einolf, C. J. (2010). Does extensivity form part of the altruistic personality? An empirical test of Oliner and Oliner's theory. Social Science Research, 39(1), 142-151. http://dx.doi. org/10.1016/j.ssresearch.2009.02.003

Flick, U. (2006). Introducción a la investigación cualitativa. Morata.

Fussangel, K. (2008). Subjektive theorien von lehrkräften zur cooperation. Eine analyse der zusammenarbeit von lehrerinnen und lehrern in lerngemeinschaften (Tesis doctoral). Universidad de Wuppertal, Wuppertal. http://d-nb.info/994090838/34

Garaigordobil, M. (2014). Conducta prosocial: El papel de la cultura, la familia, la escuela y la personalidad. RMIP, 6(2), 146-157. http://www.sc.ehu.es/ptwgalam/art completo/2014/C\%20Prosocial\%20RMIP\%202014.pdf

Hirschberger, G., Ein-dor, T. y Almakias, S. (2008). The self-protective altruist:Terror management and the ambivalent nature of prosocial behavior. Personality and Social Psychology Bulletin, 34(5), 666-78. https://doi.org/10.1177/0146167207313933

Inglés, C. J., Martínez-González, A. E. y García-Fernández, J. M. (2013). Conducta prosocial y estrategias de aprendizaje en una muestra de estudiantes españoles de Educación Secundaria Obligatoria. European Journal of Education and Psychology, 6(1), 33-53. https:// doi.org/10.30552/ejep.v6i1.88

Khalid, U. (Noviembre, 2014). Relationship between Self-Efficacy and Academic Motivation. International Conference on Economics, Education and Humanities. Bali, Indonesia. http:// icehm.org/upload/8296ED1214132.pdf

Lemos, V. N. y Richaud de Minzi, M. C. (2010). Construcción de un instrumento para evaluar el razonamiento prosocial en niños de 7 y 8 años: Una versión pictórica. Universitas Psychologica, 9(3), 879-891. https://doi.org/10.11144/Javeriana.upsy9-3.cier

López, M. J., Garrido, V., Rodríguez, F. J. y Paíno, S. G. (2002). Jóvenes y competencia social: Un programa de intervención. Psicothema, 14(supl.), 155-163. http://www.psicothema.com/ pdf/3486.pdf

Martínez, A. E., Inglés, C., Piqueras, J. A. y Oblitas, L. A. (2010). Papel de la conducta prosocial y de las relaciones sociales en el bienestar psíquico y físico del adolescente. Avances en Psicología Latinoamericana, 28(1), 74-84. http://www.scielo.org.co/pdf/apl/v28n1/ v28n1a7.pdf

Mestre, V., Samper, P., Tur, A., Cortés, M. y Nácher, M. (2006). Conducta prosocial y procesos psicológicos implicados: Un estudio longitudinal en la adolescencia. Revista Mexicana de Psicología, 23(2), 203-215. http://www.redalyc.org/pdf/2430/243020649006.pdf 
http://doi.org/10.15359/ree.24-2.8

ROR: https://ror.org/01t466c14 Universidad Nacional, Costa Rica

http://www.una.ac.cr/educare

educare@una.cr

Miranda, L. M. (2013). Cultura ambiental: Un estudio desde las dimensiones de valor, creencias, actitudes y comportamientos ambientales. Producción + Limpia, 8(2), 94-105. http://www. scielo.org.co/pdf/pml/v8n2/v8n2a10.pdf

Oyserman, D. (2015). Identity-based motivation. En R. A. Scott, M. Buchmann y S. M. Kosslyn (Eds.), Emerging trends in the social and behavioral Sciences, Hoboken, NJ: John Wiley \& Sons. https://doi.org/10.1002/9781118900772.etrds0171

Pool, Y. P. (2015). Finalidad de la educación: Convivir con otros, una aproximación del aporte de la psicología prosocial a la convivencia escolar en Chile (Tesis de pregrado). Universidad de Chile, Santiago. http://repositorio.uchile.cl/handle/2250/136676

Redondo, J., Ingles, C. y García-Fernández, J. M. (2014). Conducta prosocial y autoatribuciones académicas en educación secundaria obligatoria. Anales de Psicología, 30(2), 482-489. https://doi.org/10.6018/analesps.30.2.148331

Redondo, J., Rueda, S. y Amado, C. (2013). Conducta prosocial: Una alternativa a las conductas agresivas. Revista Investigium IRE: Ciencias Sociales y Humanas, 4(1), 234-247. http:// investigiumire.iucesmag.edu.co/index.php/ire/article/viewFile/56/55

Ruiz,M.R.(2005).Estudioeintervenciónenlaconductaprosocial-altruista(Tesisdoctoral).Universidad de Córdova, Argentina. https:/helvia.uco.es/bitstream/handle/10396/262/13217562. pdf? sequence $=1 \ldots y$

Smith, C., Hofer, J., Guillespie, M., Solomon, M. y Rowe, K. (2009). How teachers change. A study of profesional development in adult education. New York: Nova Science Publishers.

Schonert-Reichl, K. A., Smith, V., Zaidman-Zait, A., y Hertzman, C. (2012). Promoting children's prosocial behaviors in school: Impact of the "Roots of empathy" program on the social and emotional competence of school-aged children. School Mental Health, 4(1), 1-21. https:// doi.org/10.1007/s12310-011-9064-7

van den Bos, W., Vahl, P., Güroğlu, B., van Nunspeet, F., Colins, O., Markus, M., Rombouts, S. A. R. B., van der Wee, N., Vermeiren, R., Crone, E. A. (2014). Neural correlates of social decisionmaking in severely antisocial adolescents. Social Cognitive and Affective Neuroscience, 9(12), 2059-2066. https://doi.org/10.1093/scan/nsu003

Waeytens, K., Lens, W. y Vandenberghe, R. (2002). 'Learning to learn': Teachers' conceptions of their supporting role. Learning and Instruction, 12(3), 305-322. https://doi.org/10.1016/ S0959-4752(01)00024-X

Warneken, W., Lohse, K., Melis, A. P. y Tomasello, M. (2011). Young children share the spoils after collaboration. Psychological Science, 22(2), 267-273. https://doi. org/10.1177/0956797610395392 